Supporting Information For

\title{
Simultaneous Monitoring of Discrete Binding Events Using Dual-Acceptor Terbium-Based LRET
}

\author{
Kevin R. Kupcho, Deborah K. Stafslien, Therese DeRosier, Tina M. Hallis, \\ Mary Szatkowski Ozers and Kurt W. Vogel ${ }^{*}$ \\ Invitrogen Discovery Sciences, 501 Charmany Dr, Madison, WI, 53719.
}

\section{Materials and Methods}

Fluorescein-labeled CBP-1 was synthesized by Anaspec (San Jose, CA). A peptide corresponding to NCoR-ID2 was synthesized by Anaspec with a reactive cysteine at the $\mathrm{N}$-terminus, labeled with Alexa Fluor $^{\circledR} 633$ (AF633)-maleimide (Invitrogen/Molecular Probes; Eugene, OR), and purified by HPLC. Solutions of fluorescein-CBP-1 were quantified by $\varepsilon_{493}=73,000 \mathrm{M}^{-1} \mathrm{~cm}^{-1}$ in $0.1 \mathrm{~N} \mathrm{NaOH}$. Solutions of AF633-NCoR-ID2 were quantified by $\varepsilon_{622}=110,000 \mathrm{M}^{-1} \mathrm{~cm}^{-1}$ in PBS or $50 \mathrm{mM}$ HEPES, pH 7.5. The peptide sequences are shown below:

Fluorescein-CBP-1

Fluorescein-AASKHKQLSELLRGGSGSS

AF633-NCoR-ID2

AF633-CNLGLEDIIRKALMG

Rosiglitazone was from Alexis Biochemical (San Diego, CA). L165041 and GW1929 were from Tocris (Ellisville, MO). T0070907 was from Calbiochem (San Diego, CA). Terbium (Tb) anti-GST antibody, nuclear receptors, assay buffers and the cell line and reagents used for the cell-based PPAR $\delta$ assay were from Invitrogen Corporation (Madison, WI).

\section{Ligand titrations and determination of $\mathrm{EC}_{50}$ values for peptide recruitment and displacement}

For all assays, ligands were first prepared at 100X of the desired final concentration in DMSO by serially diluting the maximum concentration of ligand to be used in DMSO in a 96-well polypropylene plate from Nalge Nunc (Rochester, NY) or 384-well polypropylene plate from Costar/Corning (Corning, $\mathrm{NY}$ ) in order to maintain ligand solubility. The ligands were then diluted to a $2 X$ concentration in assay buffer in the same polypropylene plate. A $10 \mu \mathrm{L}$ aliquot of the $2 \mathrm{X}$ ligand dilutions was then added to each well of the 384-well black assay plate. Five $\mu \mathrm{L}$ of the respective GST-tagged receptor and $5 \mu \mathrm{L}$ of a solution containing peptides and Tb-anti GST antibody were then added to each assay well, for a 
final assay volume of $20 \mu \mathrm{L}$. Each receptor was used at a final concentration of $5 \mathrm{nM}$ in the assay. PPAR $\alpha$ assays contained 125 nM Fluorescein-CBP-1 and 250 nM AF633-NCoR-ID2. PPAR $\delta$ and PPAR $\gamma$ assays contained $100 \mathrm{nM}$ or $125 \mathrm{nM}$ of each labeled peptide, respectively. PPAR $\alpha$ and PPAR $\gamma$ assays contained $5 \mathrm{nM}$ Tb-anti GST antibody, and PPAR $\delta$ assays contained $10 \mathrm{nM}$ antibody. The final DMSO concentration in all assay wells was $1 \%$. The plates was mixed gently for 30 seconds on a plate shaker, incubated at room temperature for 4 to 5 hours, and evaluated in a plate reader for LRET detection.

\section{Measurement of ligand-dependent and ligand independent association of peptides with receptors}

To $5 \mu \mathrm{L}$ of ligand in $4 \%$ DMSO at 4 -fold the final concentration to be tested was added $5 \mu \mathrm{L}$ of receptor, $5 \mu \mathrm{L}$ of antibody, and $5 \mu \mathrm{L}$ of a serial dilution of either Fluorescein-CBP1 or AF633-NCoRID2 peptide. Buffer composition and receptor, antibody, and peptide concentrations were as described above for the ligand-dependent peptide recruitment/displacement experiments. Wells used to measure ligand-independent association of peptide lacked ligand, and wells used to measure background FRET due to diffusion-enhanced FRET between Tb-labeled antibody and fluorophore-labeled peptide lacked ligand as and receptor. The plates were mixed gently for 30 seconds on a plate shaker, incubated at room temperature for 4 to 5 hours, and evaluated in a plate reader for LRET detection.

\section{Measurement of LRET}

Experiments to measure $\mathrm{EC}_{50}$ values for ligand-dependent association and dissociation of peptides were performed using a Tecan Ultra plate reader (Tecan, Durham, NC). The terbium donor was excited using a $340 \mathrm{~nm}$ excitation filter with a $30 \mathrm{~nm}$ bandwidth. The fluorescein signal was measured using a $520 \mathrm{~nm}$ filter with a $25 \mathrm{~nm}$ bandwidth, the AF633 signal was measured with a $665 \mathrm{~nm}$ filter with a $10 \mathrm{~nm}$ bandwidth, and the terbium donor signal was measured using a $495 \mathrm{~nm}$ filter with a $10 \mathrm{~nm}$ bandwidth. TR (time resolved) -FRET values (emission ratios) were calculated as the ratio of raw acceptor to donor intensities measured over a $200 \mu$ s signal integration time following a $100 \mu$ s post- 
excitation delay and averaged over 10 excitations (flashes) per well. No background subtraction or crosstalk correction was necessary. The "fluorescein" dichroic mirror (Tecan) was used to measure both donor and fluorescein acceptor signals, and the "Cy5" dichroic mirror was used to measure emission from AF633. Binding curves were generated by plotting the emission ratios against the log of the ligand concentration. The data were fit using non-linear regression with an equation for sigmoidal dose response (variable slope) in GraphPad ${ }^{\mathrm{TM}}$ Prism $^{\circledR}$ (GraphPad Software, Inc, San Diego CA). Experiments to show increased or decreased affinity of peptides for ligand bound receptors were performed on a BMG Pherastar plate reader using the LanthaScreen filter module to measure FRET to fluorescein labeled peptides and the HTRF filter module to measure FRET to AF633-labeled peptides.

It should be noted that because energy transfer from excited $\mathrm{Tb}^{3+}$ to AF633 depopulates the excited state of $\mathrm{Tb}^{3+}$, it therefore results in a decrease of emission from all four $\mathrm{Tb}^{3+}$ emission peaks, since all of these emissions originate from the same excited state of $\mathrm{Tb}^{3+}$. For convenience, the 495 emission peak was chosen as a reference donor signal when measuring energy transfer to either fluorescein or AF633. Additionally, because not all $\mathrm{Tb}^{3+}$ chelates present in the system participate in FRET (either because the $\mathrm{Tb}^{3+}$-labeled antibody is not fully saturated with receptor, or because not all $\mathrm{Tb}^{3+}$ chelates are positioned for efficient FRET), the absolute decrease in the donor signal is negligible relative to the absolute increase in the acceptor signal.

\section{Discussion}

The multiplexed measurement of ligand dependent association or dissociation of peptides with a receptor was compared to measurements made with only a single peptide present, with the results shown in Figure S1 and tabulated in Tables S1 and S2. There was generally close correlation between the measurements made in the multiplexed experiments as compared to measurements made with only a single peptide present. It should be noted though that an exact correlation is not expected, since in the presence of multiple peptides the equilibria will be shifted in one direction or 
another depending on ligand-dependent and ligand independent associations between the receptor and the two peptides present (Scheme S1).
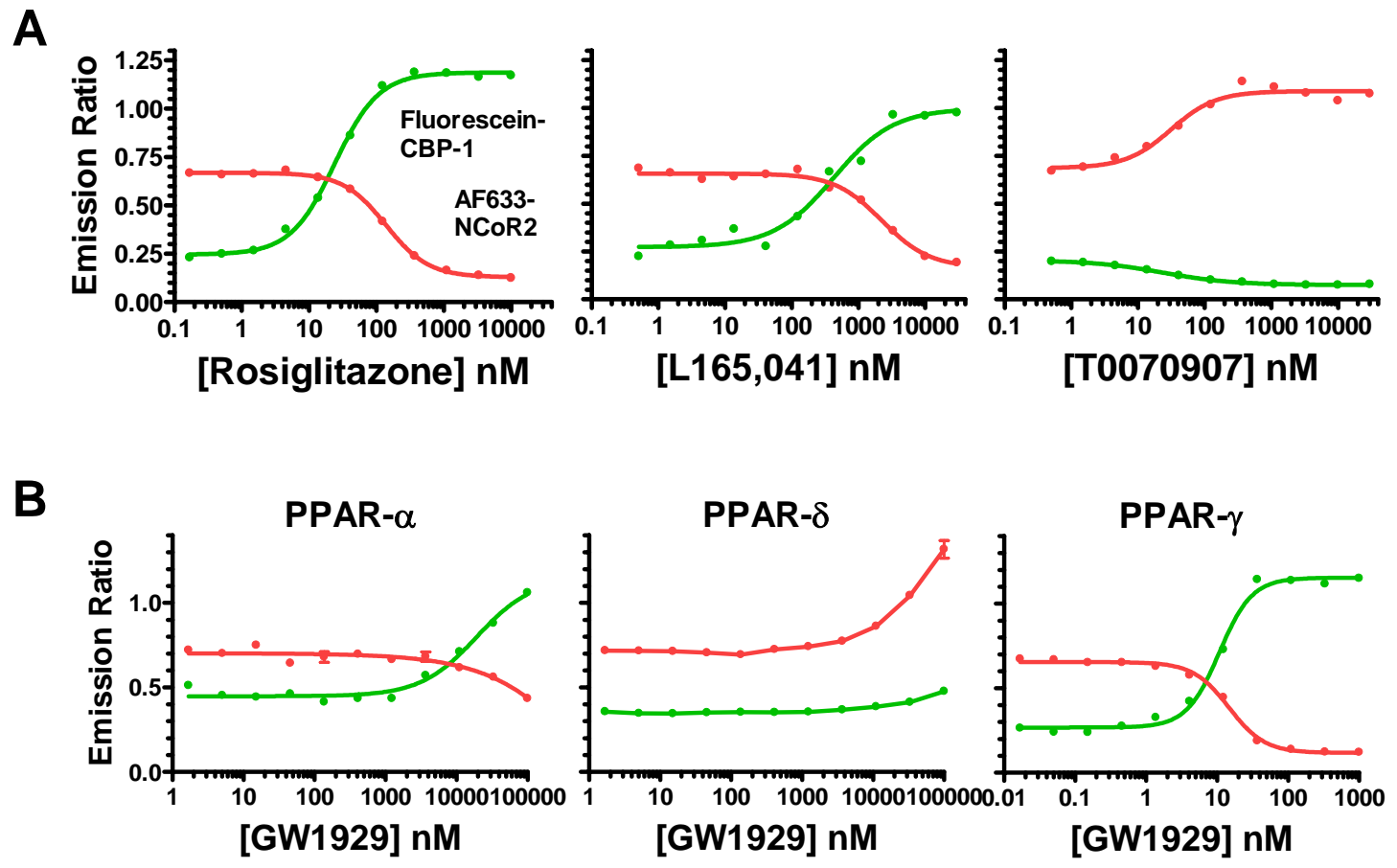

Figure S1: Individual monitoring of ligand-dependent and ligand-independent association of coregulator peptides and receptors. Coactivator and corepressor peptide interactions with receptor were measured individually (with only one peptide present) but are shown on the same graph for comparison purposes with Figure 2 of the manuscript. (A) Individual monitoring of ligand-dependent and ligand-independent association of fluorescein-labeled coactivator $\left(\mathrm{CBP}-1,{ }^{\circ}\right)$ and AF633-labeled corepressor (NCoR-ID2, ${ }^{\bullet}$ ) derived peptides with PPAR $\gamma$. (B) Differential response of PPAR $\alpha, \delta$ and $\gamma$ to GW1929. Each datapoint is the average of 4 replicate wells. Error bars marking standard deviations are generally smaller than the symbols used to mark each datapoint.

\section{FI-CBP-1}

Ligand

Rosiglitazon

e

L165041

T0070907 w/ AF633-

Fl-CBP-1 Alone $\quad$ NCoR-ID2

$24 \mathrm{nM}$

$420 \mathrm{nM}$

$24 \mathrm{nM}$
$53 \mathrm{nM}$

$1.03 \mu \mathrm{M}$

$102 \mathrm{nM}$
AF633-NCoR-ID2

AF633-NCoR-

ID2 Alone

$138 \mathrm{nM}$

$2.2 \mu \mathrm{M}$

$31 \mathrm{nM}$ $\underline{w / F I-C B P-1}$

$74 \mathrm{nM}$

$1.23 \mu \mathrm{M}$

$25 \mathrm{nM}$ 
Table S1: $\mathrm{EC}_{50}$ values for ligand dependent association/dissociation of FI-CBP-1 and AF633-NCoRID2 with PPAR $\gamma$ as measured in a single-peptide or a multiplexed format.

\begin{tabular}{|c|c|c|c|c|}
\hline \multirow[b]{2}{*}{ Receptor } & \multicolumn{2}{|c|}{ FI-CBP-1 } & \multicolumn{2}{|c|}{ AF633-NCoR-ID2 } \\
\hline & Fl-CBP-1 Alone & $\begin{array}{l}\text { w/ AF633- } \\
\text { NCoR-ID2 }\end{array}$ & $\begin{array}{l}\text { AF633-NCoR- } \\
\text { ID2 Alone }\end{array}$ & w/ Fl-CBP-1 \\
\hline PPAR- $\alpha$ & $>10 \mu \mathrm{M}$ & $>10 \mu \mathrm{M}$ & $>10 \mu \mathrm{M}$ & $>10 \mu \mathrm{M}$ \\
\hline PPAR- $\delta$ & $>10 \mu \mathrm{M}$ & $>10 \mu \mathrm{M}$ & $>10 \mu \mathrm{M}$ & $>10 \mu \mathrm{M}$ \\
\hline PPAR- $\gamma$ & $10.2 \mathrm{nM}$ & $5.2 \mathrm{nM}$ & $14.5 \mathrm{nM}$ & $5.8 \mathrm{nM}$ \\
\hline
\end{tabular}

Table S2: $\mathrm{EC}_{50}$ values for GW1929 dependant association/dissociation of CBP-1 and NCoR-ID2 with PPAR $\alpha, \delta$, and $\gamma$ as measured in a single-peptide or a multiplexed format.

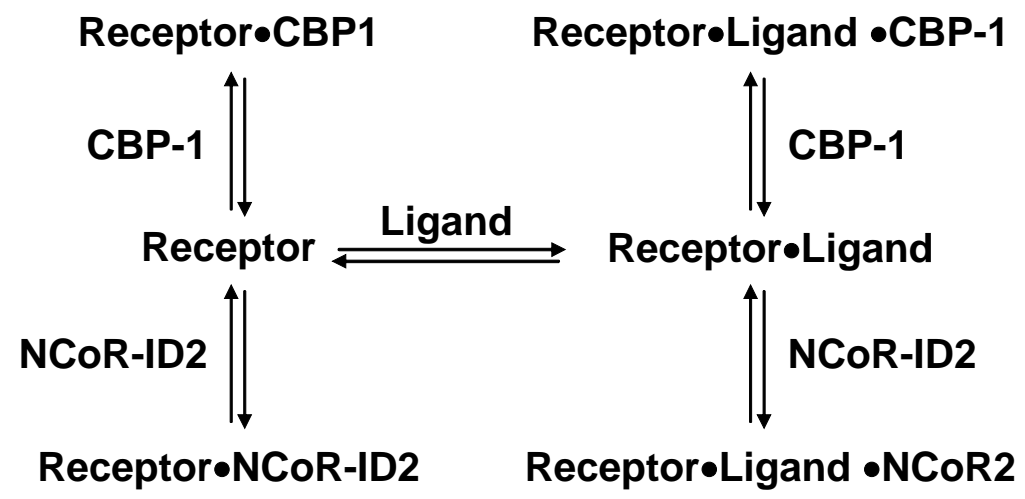

Scheme S1: Schematic of multiple equilibria involved in measuring peptide•receptor interactions. This schematic assumes that only one peptide at a time can bind to the receptor, and that ligand is only able to bind receptor that is not associated with peptide. These two assumptions are consistent with current understanding of the conformational changes that take place in nuclear receptors that allow ligands and coactivators to bind. ${ }^{1}$

The concentrations of antibody, receptors, and peptides used in this study were not extensively optimized. In general, to optimize these sorts of assays (in a non-multiplexed format) we first investigate associations between a receptor and a known ligand, either using a coactivator or corepressor peptide derived from a known coactivator or co-repressor (typically at ca. $500 \mathrm{nM}$ concentration), or against a panel of approximately 30 peptides derived from various coregulator proteins. ${ }^{2}$ In this first experiment, FRET may be measured in the presence or absence of ligand, using 
a matrix of different receptor and antibody concentrations ranging between approximately 1 and $10 \mathrm{nM}$ each, and minimal concentrations of each reagent are chosen in order to maintain a robust signal change. Following this, the peptide may be titrated against the receptor and antibody in the presence or absence of ligand, in order to determine conditions under which ligand-dependent association can be most robustly discerned from ligand-independent association. Because at high concentrations of fluorescently labeled peptide diffusion enhanced $\mathrm{FRET}^{3}$ will occur between the terbium labeled antibody and the fluorophore labeled peptide, non-ligand dependent association of peptide with receptor can be discerned from this background signal by performing the titration in the absence of receptor. Similarly configured assay optimizations can be performed to observe ligand-independent receptor-peptide associations, and ligand-dependent disruptions of these interactions. It is important to note however that the conditions chosen for the most robust ligand-dependent signal change between a given receptor and peptide combination are not necessarily the same for other ligands that will bind to that receptor to cause peptide association or dissociation.

To further confirm the ligand-dependent increases and decreases in affinity between PPARs and coactivator or corepressor peptides that were observed in this study, we directly measured peptide affinity for PPAR $\alpha, \delta$, and $\gamma$ in the presence or absence of ligands (Figure S2). NCoR-ID2 showed ligand-independent association with PPAR $\alpha$, and the affinity of this interaction was decreased in the presence of GW1929. In contrast, the affinity of CBP-1 for PPAR $\alpha$ was increased in the presence of GW1929. NCoR-ID2 showed a slight degree of ligand-independent association with PPAR $\delta$, and the affinity of this interaction was increased in the presence of GW1929. CBP-1 showed essentially no affinity for PPAR in the absence of ligand, but a slight association in the presence of GW1929. NCoR-ID2 showed ligand-independent association with PPAR $\gamma$, and the affinity of this interaction was increased in the presence of T0070907. However, at higher concentrations of NCoR-ID2 the extent of ligand-dependent and ligand independent association was indistinguishable. Finally, CBP-1 showed ligand-independent interaction with PPAR $\gamma$, and the affinity of this interaction was increased in the presence of rosiglitazone, L165041, or GW1929. It should be noted that the instrument used to 
measure emission ratios in these particular experiments was different than the instrument used to perform the ligand-titration experiments, and therefore the donor and acceptor signal intensities were on a different scale than those of the other experiments. Thus the absolute values of the Y-axes cannot be compared between these separate experiments. However, all of the observed results were consistent with the ligand titration experiments shown in Figure 2.
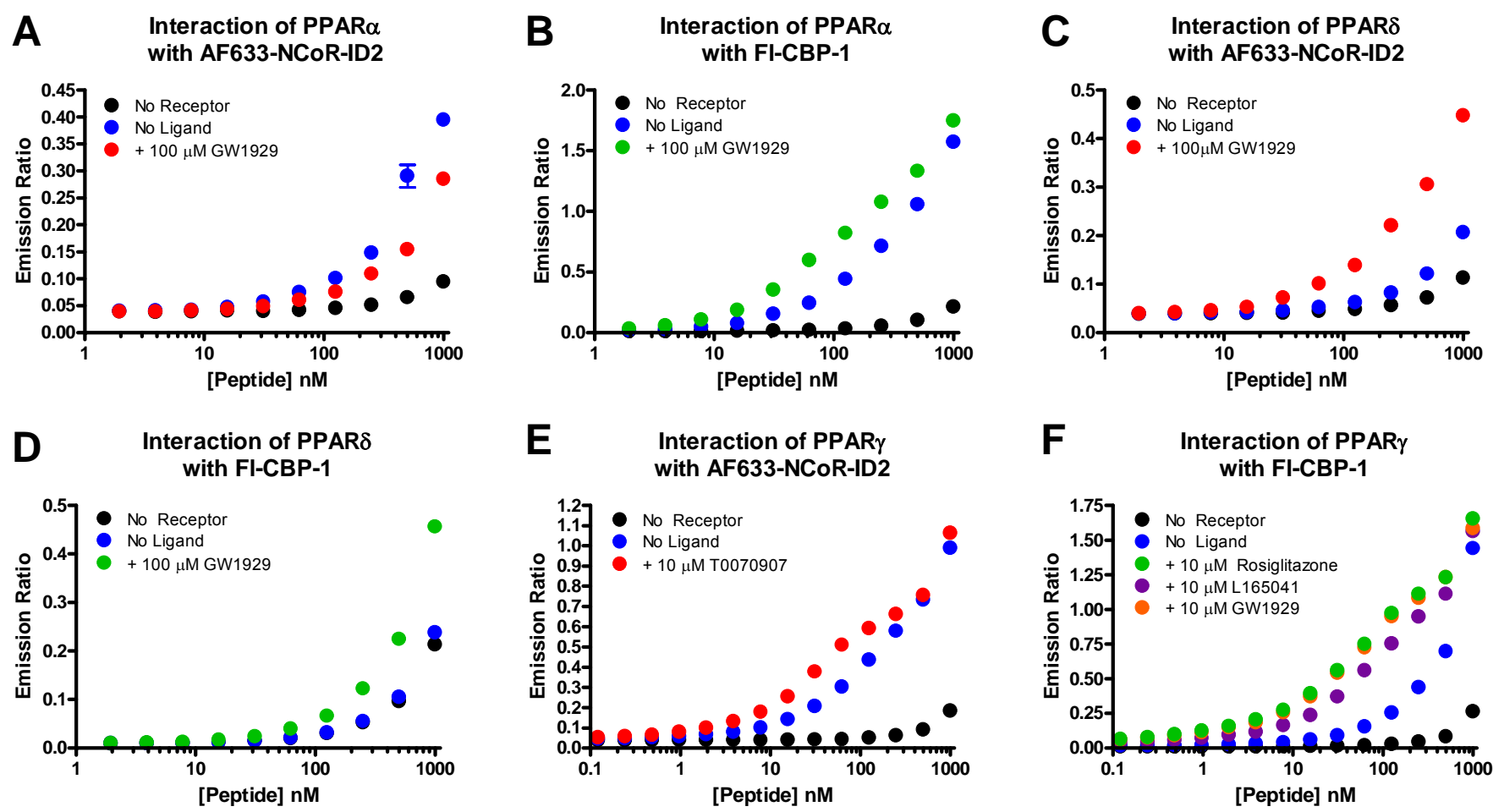

Figure S2: Ligand dependent and ligand-independent association of FI-CBP1 or AF633-NCoR-ID2 with PPAR $\alpha, \delta$, or $\gamma$. (A) NCoR-ID2 association with PPAR $\alpha$ (B) CBP-1 association PPAR $\alpha$ (C) NCoRID2 association with PPAR $\delta$ (D) CBP-1 association with PPAR $\delta$ (E) NCoR-ID2 association with PPAR $\gamma(F)$ CBP-1 association with PPAR $\gamma$. Each datapoint is the average of 3 replicate wells. Error bars marking standard deviations are generally smaller than the symbols used to mark each datapoint.

Finally, the action of GW1929 as a PPAR $\delta$ antagonist was confirmed using a commercial cell-based assay (GeneBLAzer ${ }^{\circledR}$ PPAR-UAS-bla HEK 293T Cells, Invitrogen) that uses expression of a $\beta$ lactamase reporter gene $e^{4}$ to measure the transactivation of a chimera of a PPAR $\delta$ ligand binding domain that is expressed as a fusion with the GAL4 DNA binding domain (Figure S3). In this 
experiment, GW1929 was shown to antagonize L165041 (agonist) induced expression of the $\beta$ lactamase reporter gene, consistent with results of our simplified biochemical assay.
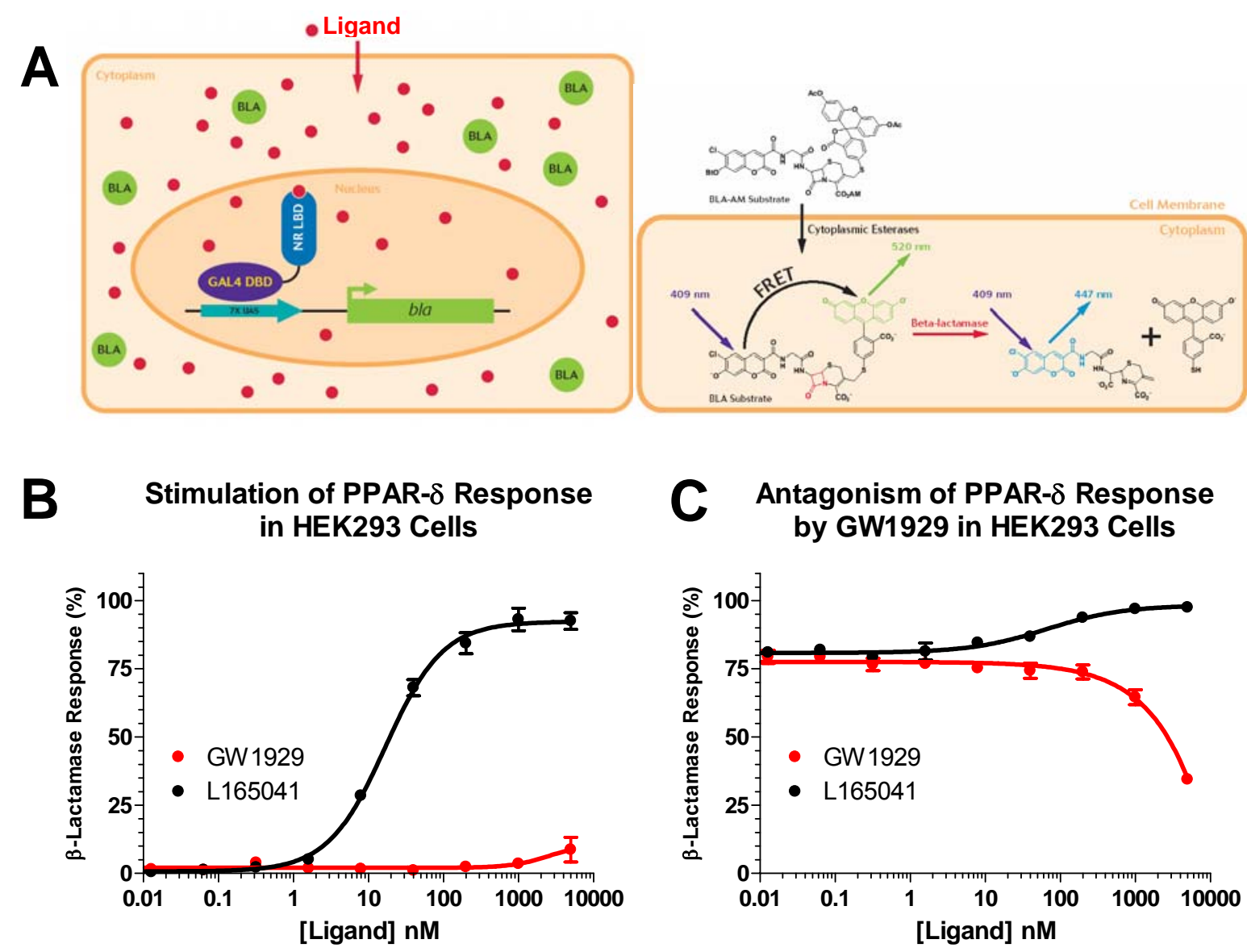

Figure S3. Confirmation that GW1929 acts as an antagonist in a cell-based reporter assay. (A) Assay Schematic. The PPAR $\delta$-GAL4 cell-based assay was developed by stably expressing the GAL4DNA binding domain (DBD) fused to the PPAR $\delta$ ligand binding domain (LBD) in HEK293 cells engineered with $\beta$-lactamase (BLA) cDNA under transcriptional control of an Upstream Activator Sequence (UAS). The GAL4 yeast transcription factor DBD binds to its UAS target sequence, and upon binding of agonist to the PPAR $\delta$ LBD portion of the GAL4 fusion, cofactors are recruited that result in initiation of BLA transcription. The expression of BLA is detected using a cell permeable FRET based substrate. The intact substrate undergoes efficient FRET, which produces green fluorescence. Production of BLA enzyme upon treatment of cells with a PPAR $\delta$ agonist results in cleavage of the substrate by BLA which results in the loss of FRET (blue fluorescence). (B) In the absence of ligand, $\beta$-lactamase is not expressed, but is expressed upon treatment of the cells with the known PPAR $\delta$ agonist L165041. (C) When cells are treated with a sub-maximal concentration of $\mathrm{L} 165041(60 \mathrm{nM})$ the $\beta$-lactamase response is decreased at high concentrations of GW1929. 


\section{Acknowledgements}

The authors thank Jennifer Wilkinson for generating the cell-based assay data in Figure S3.

\section{Complete citations for references 13 and 15}

13. Berger, J.; Leibowitz, M. D.; Doebber, T. W.; Elbrecht, A.; Zhang, B.; Zhou, G.; Biswas, C.; Cullinan, C. A.; Hayes, N. S.; Li, Y.; Tanen, M.; Ventre, J.; Wu, M. S.; Berger, G. D.; Mosley, R.; Marquis, R.; Santini, C.; Sahoo, S. P.; Tolman, R. L.; Smith, R. G.; Moller, D. E. J Biol Chem 1999, 274, (10), 6718-25.

15. Brown, K. K.; Henke, B. R.; Blanchard, S. G.; Cobb, J. E.; Mook, R.; Kaldor, I.; Kliewer, S. A.; Lehmann, J. M.; Lenhard, J. M.; Harrington, W. W.; Novak, P. J.; Faison, W.; Binz, J. G.; Hashim, M. A.; Oliver, W. O.; Brown, H. R.; Parks, D. J.; Plunket, K. D.; Tong, W. Q.; Menius, J. A.; Adkison, K.; Noble, S. A.; Willson, T. M. Diabetes 1999, 48, (7), 1415-24.

\section{Supplemental References}

1. (a) Bourguet, W.; Germain, P.; Gronemeyer, H. Trends Pharmacol Sci 2000, 21, (10), 381-8

(b) Gronemeyer, H.; Gustafsson, J. A.; Laudet, V. Nat Rev Drug Discov 2004, 3, (11), 950-64.

2. Gowda, K.; Marks, B. D.; Zielinski, T. K.; Ozers, M. S. Anal Biochem 2006, 357, (1), 105-15.

3. $\quad$ Stryer, L.; Thomas, D. D.; Meares, C. F. Annu Rev Biophys Bioeng 1982, 11, 203-22.

4. Zlokarnik, G.; Negulescu, P. A.; Knapp, T. E.; Mere, L.; Burres, N.; Feng, L.; Whitney, M.; Roemer, K.; Tsien, R. Y. Science 1998, 279, (5347), 84-8. 